\title{
Estrés laboral en personal asistencial de cuatro instituciones de salud nivel III de Cali, Colombia
}

\author{
Occupational stress in healthcare staff of four level III health institutions of Cali in Colombia
}

Kewy Sarsosa-Prowesk ${ }^{1 *}$ orcid.org/0000-0002-8906-3651

Víctor Hugo Charria-Ortiz' orcid.org/0000-0002-9138-7460

1 Facultad de Humanidades y Ciencias Sociales, Departamento de Ciencias Sociales, Grupo de investigación Bienestar, Trabajo, Cultura y Sociedad (BITACUS). Pontificia Universidad Javeriana. Cali, Colombia

Fecha de recepción: Marzo 27 - $2017 \quad$ Fecha de revisión: Noviembre 10 - $2017 \quad$ Fecha de aceptación: Diciembre 21 - 2017

Sarsosa-Prowesk K, Charria-Ortiz VH. Estrés laboral en personal asistencial de cuatro instituciones de salud nivel III de Cali, Colombia. Univ. Salud. 2018;20(1):44-52. DOI: http://dx.doi.org/10.22267/rus.182001.108

\section{Resumen}

Introducción: El estrés entendido como el conjunto de respuestas fisiológicas, comportamentales, emocionales y cognoscitivas, que resultan en el proceso de adaptación frente a las demandas derivadas de las condiciones de trabajo ante las cuales las personas evalúan que sus recursos de afrontamiento son insuficientes. Objetivo: Identificar el nivel de estrés laboral en personal con cargos asistenciales de cuatro instituciones de salud nivel III de la ciudad de CaliColombia. Materiales y métodos: El estudio fue de tipo descriptivo, con diseño transversal. Se aplicó el cuestionario para la evaluación del estrés tercera versión del Ministerio de la Protección Social a una muestra de 595 participantes, con cargo asistencial que implica la atención sanitaria al paciente, en procedimientos de alta complejidad como cirugía, hospitalización, urgencias, cuidados intensivos. Resultados: El personal con cargo asistencial de las cuatro instituciones de salud nivel III presenta nivel alto de estrés laboral, concretamente en los síntomas fisiológicos, e intelectuales y laborales. Conclusiones: La prevalencia de estrés laboral en el personal asistencial puede asociarse con las características de rol, las condiciones laborales y la exposición permanente a enfermedades contagiosas propias del contexto de las instituciones de salud.

Palabras clave: Estrés laboral; agentes comunitarios de salud; psicología; instituciones de salud. (Fuente: DeCS, Bireme).

\begin{abstract}
Introduction: Stress is understood as the set of physiological, behavioral, emotional and cognitive responses, which result in the process of adaptation to the demands derived from the working conditions, before which people evaluate that their coping resources are insufficient. Objective: To identify the level of occupational stress in staff with care positions of four level III health institutions in the city of Cali, Colombia. Materials and methods: A descriptive study with transversal design was made. The third version stress assessment questionnaire was applied Ministry of Social Protection. The sample was 595 participants with care charge, which involves the patient's health care in procedures of high complexity such as surgery, hospitalization, emergencies, intensive care. Results: The staff with healthcare charge of the four level III health institutions presents high level of occupational stress, particularly in the physiological, intellectual and labor symptoms. Conclusions: The prevalence of occupational stress in health care personnel can be associated with the characteristics of role, working conditions and the permanent exposure to communicable diseases inherent in the context of healthcare institutions.
\end{abstract}

Keywords: Burnout, professional; community health workers; psychology; health facilities. (Source: DeCS, Bireme). 


\section{Introducción}

El estrés laboral es una enfermedad de origen laboral reportada por el Ministerio del Trabajo en la tabla de Enfermedades Laborales, perteneciente al grupo IV denominado Trastornos Mentales y del Comportamiento, por lo cual es una amenaza para la salud mental, que puede generar enfermedades cardiovasculares y accidentes de trabajo ${ }^{(1)}$. Según los resultados de la Segunda Encuesta Nacional de Condiciones de Seguridad y Salud en el Trabajo en el Sistema General de Riesgos Laborales de Colombia(2), se evidenció la prevalencia de estrés por exposición a factores de riesgo psicosocial, principalmente en los trabajadores que atienden público, por ser vulnerables a la violencia verbal de un tercero. Esta encuesta además informa que algunas de las condiciones que más generan estrés laboral en la población colombiana, son: posturas que deben adoptar las personas para realizar su trabajo y que producen cansancio o dolor físico, el ritmo de trabajo que conlleva a falta de tiempo para completar las tareas, horario de trabajo, falta de claridad sobre su rol o función, desajustes entre las exigencias del trabajo y la competencia laboral del empleado, trabajo excesivamente exigente, falta de apoyo de los jefes y compañeros, sobrecarga de trabajo, poca recompensa $y$ oportunidades de ascensos o desarrollo profesional, comunicación ineficaz y el cambio organizacional no planeado. Estas condiciones, tienen efecto psicológico, físico y en las relaciones sociales en el entorno laboral(2).

El 60\% del personal asistencial del sector salud, tiene turnos diarios de trabajo entre 9 y 12 horas $\mathrm{y}$ enfrentan situaciones en sus funciones tales como atender personas gravemente enfermas, tomar decisiones que implican salvar vidas o inevitablemente enfrentar la muerte(2). Las condiciones de trabajo del sector salud en Colombia están regidas por la Ley 100 de 1993(3), la necesidad de la atención médica de 24 horas y la normativa de los turnos rotativos han desencadenado el fenómeno del pluriempleo y prevalencia de estrés laboral.

El estrés laboral se define como el grupo de reacciones de naturaleza emocional, comportamental y psicológica generadas por las demandas del ambiente para las cuales el individuo tiene una incapacidad de

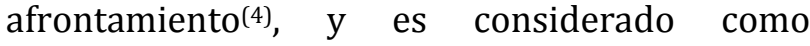
padecimiento laboral de alta prevalencia en el mundo moderno, que genera un desequilibrio causado por la insuficiente competencia de generar una respuesta viable ante una exigencia del entorno, afectando la salud y bienestar del trabajador, lo cual es generador de enfermedades en el ámbito laboral que parte de la experiencia y percepciones del individuo en su interrelación con las condiciones laborales, causando afecciones o síntomas nocivos en la salud(5-7).

El estrés laboral desencadena aumento de ausentismo, baja productividad, alta rotación, presencia de enfermedades y accidentes laborales, entre otros $(8,9)$. Entre un 50\% y un $60 \%$ del ausentismo laboral está relacionado con el estrés laboral(5). Diferentes investigaciones han evidenciado la relación entre manifestaciones de estrés laboral y aspectos como el tipo de servicio que presta la organización, la carga mental, la duración de la jornada laboral y la frustración ante la tarea(10-12).

Algunos autores como Atalaya(8); Crespo y Labrador(13); y Sosa(14), coinciden en definir el estrés como el desajuste entre una respuesta adaptativa y demandas externas que son percibidas como amenazantes por sobrepasar las capacidades tanto físicas como mentales del individuo. En consecuencia, el estrés en el trabajo es comprendido como las reacciones físicas y emocionales que ocurren cuando las exigencias del trabajo no igualan las capacidades del trabajador; es así, como la tensión generada en el proceso de estrés, está mediada por las características de personalidad y/o por procesos psicológicos implicados, según el nivel de intensidad, puede ser positiva para mejorar la capacidad de respuesta, o negativa cuando no permite una respuesta adecuada a la demanda del entorno, desencadenando sufrimiento psicológico y enfermedades(14).

Se distinguen tres clasificaciones para el estrés laboral; la primera, explica el estrés como el grupo de fuerzas externas que logran producir efectos 
transitorios o permanentes. La segunda, define el estrés como las múltiples respuestas de carácter fisiológico o psicológico que se pueden suscitar de determinados estímulos. Y la tercera, describe al estrés desde las consecuencias psicológicas y físicas originadas por procesos perceptuales ${ }^{(15)}$.

El estrés se concibe, como un proceso de desbalance entre las exigencias de un evento y los recursos con los que cuenta el individuo para hacerle frente ${ }^{(16,17)}$. Se encauza el estrés laboral, sobre la actividad productiva del trabajo, donde no se logra satisfacer las necesidades materiales o simbólicas del individuo (18). Es así, como cada vez es más frecuente que las personas padezcan estrés laboral, relacionándolo con su salud laboral(1,19-21).

En el sector de la salud, diversas investigaciones, revelan que los trabajadores asistenciales presentan una prevalencia entre un 33,9\% y un $65 \%$ de estrés laboral, asociado con síntomas de carácter emocional y/o fisiológico, poniendo de manifiesto que las profesiones en el ámbito de la salud, son potencialmente estresantes, por las características de rol, la falta de definición de tareas, la sobre carga laboral y condiciones laborales, además de una exposición permanente a enfermedades contagiosas(6,22-24).

Un estudio en Colombia reporta que entre el 20\% y 33\% de los profesionales asistenciales médicos, presentan niveles de estrés laboral altos que afectan principalmente los rasgos fisiológicos y emocionales de la persona, evidenciando como consecuencia, pérdida de interés por el trabajo, baja motivación por las tareas, ansiedad, cansancio emocional, fatiga, insomnio, úlceras y despersonalización(25). El sector salud es una población en riesgo de sufrir diversas enfermedades profesionales y estar expuesta a factores psicosociales de riesgo, dadas las características de su actividad laboral, de las cuales no se puede abstraer(27-29).

Las instituciones de salud cuya misión consiste en velar por el cuidado y bienestar físico y mental de los pacientes, debe procurar que sus trabajadores encuentren las mismas condiciones para garantizar un buen funcionamiento del sistema de salud un servicio de alta calidad generado en gran medida por los conocimientos, habilidades, competencias y motivaciones de los trabajadores de la salud(30-33).

El contexto laboral simboliza la forma de expresión del hombre en el ámbito social y económico, sus reportorios laborales le otorgan significado emocional y cognoscitivo al hombre ${ }^{34-}$ 36); lo cual implica que el trabajo como actividad humana no solo tiene un sentido productivo, sino que también da un estatus e identidad social, como una persona económicamente activa contribuyendo a la representación social del trabajo(36,37). En tal sentido, Ancinas( ${ }^{(38)}$ manifiesta que: "cuando se trabaja con personas, y específicamente con el sufrimiento humano, es común encontrar malestar en los trabajadores, cuyo trabajo implica tratar con situaciones estresantes y emocionalmente demandantes".

El estrés laboral es originado en gran parte por el sentimiento de los trabajadores asistenciales de ser responsables de la vida humana y la incertidumbre que conlleva el diagnóstico y el tratamiento de cada paciente. Así como también, la exposición a los conflictos éticos y legales particulares del sector salud en los que está inmerso el personal asistencial(39). Además, las jornadas de trabajo extenuantes, la sobrecarga laboral y el pluriempleo caracterizado por los turnos rotarios, son desencadenantes del ausentismo laboral por causa médica $\mathrm{y}$ disminución del desempeño laboral(40).

Esta investigación cobra gran relevancia al identificar el nivel de estrés laboral en personal asistencial, de cuatro instituciones del sector salud nivel III de la ciudad de Cali, Colombia que permite profundizar en la descripción y conocimiento de esta problemática, la cual hoy en día tiene gran reconocimiento en el campo de la salud laboral por sus implicaciones en el bienestar y la salud de los trabajadores(41). Particularmente, incursiona en la identificación de un sector que no había sido caracterizado previamente en la ciudad, brindando un insumo que puede ser utilizado para mejorar las condiciones de trabajo del sector laboral específico. Además, aporta evidencias de la 
aplicación de un instrumento validado en el país y explora su utilización y su adaptabilidad para el sector de la salud.

El personal asistencial, es una población que está expuesta a situaciones de trabajo exigente y estresante al manejar casos complejos de la salud. Es pertinente estudiar el estrés por cuanto en Colombia(42), es obligación de las organizaciones identificar, evaluar, prevenir, intervenir $\mathrm{y}$ monitorear los factores de riesgo psicosocial y la determinación de patologías causadas por el estrés laboral, retomando el modelo dinámico propuesto por Villalobos(43), al señalar que los factores psicosociales en el trabajo deben ser comprendidos de manera integral que contemple los posibles elementos y relaciones que influyen en la experiencia laboral de los trabajadores.

\section{Materiales y métodos}

La investigación corresponde a un enfoque cuantitativo, con un diseño no experimental y un estudio de tipo descriptivo de corte transversal. La muestra fue incidental, conformada por 595 participantes, con los siguientes criterios de inclusión: ocupar un cargo asistencial (médicos, enfermeras, auxiliares de enfermería, auxiliares de laboratorio, entre otros, que tienen como funciones la atención sanitaria, es decir, el contacto directo con los pacientes y con proveer un servicio en salud). Estar vinculados laboralmente en cuatro clínicas nivel III de la ciudad de Cali, Colombia (el nivel III corresponde a instituciones que cuentan con equipo médico de alta tecnología para realizar procedimientos de alta complejidad: cirugía especializada, hospitalización, urgencias, cuidados intensivos y ofrecen atención las 24 horas).

Se aplicó el cuestionario para la evaluación del estrés en su tercera versión del Ministerio de la Protección Social(44). El cual fue validado con una muestra de 5.421 personas de diferentes regiones del país. A este instrumento se le realizó también validación de contenido con trabajadores de servicios médicos asistenciales y profesionales de salud ocupacional, con quienes se establecieron los síntomas comunes relacionados con estrés. La consistencia interna del cuestionario, se estimó a través del Alfa de Cronbach, con un valor de 0,88 que corresponde a una muy buena confiablidad. El propósito del cuestionario es identificar las causas de la presencia de estrés, con un total de 31 ítems, distribuidos en 4 síntomas, que se describen a continuación: síntomas fisiológicos (8 ítems), síntomas de comportamiento social (4 ítems), síntomas intelectuales y laborales (10 ítems) y síntomas psicoemocionales (9 ítems).

- Síntomas fisiológicos, se indagan por medio de: dolores en el cuello y espalda o tensión muscular; problemas gastrointestinales, úlcera péptica, acidez, problemas digestivos o del colon; problemas respiratorios; dolor de cabeza; trastornos del sueño como somnolencia durante el día o desvelo en la noche; palpitaciones en el pecho o problemas cardíacos; cambios fuertes del apetito y problemas relacionados con la función de los órganos genitales (impotencia, frigidez).

- Síntomas de comportamiento social, se indagan por medio de: dificultades en las relaciones familiares; para permanecer quieto o para iniciar actividades; dificultad en las relaciones con otras personas, sensación de aislamiento y desinterés.

- Síntomas intelectuales y laborales, se indagan por medio de: sentimiento de sobrecarga de trabajo; dificultad para concentrarse, olvidos frecuentes; aumento en el número de accidentes de trabajo; sentimiento de frustración, de no haber hecho lo que se quería en la vida; cansancio, tedio o desgano; disminución del rendimiento en el trabajo o poca creatividad; deseo de no asistir al trabajo; bajo compromiso o poco interés con lo que se hace; dificultad para tomar decisiones y deseo de cambiar de empleo.

- Síntomas psicoemocionales, se indagan por medio de: sentimiento de soledad y miedo; sentimiento de irritabilidad, actitudes y pensamientos negativos; sentimiento de angustia, preocupación o tristeza; consumo de drogas para aliviar la tensión o los nervios; sentimientos de que "no vale nada", o " no sirve para nada"; consumo de bebidas 
alcohólicas o café o cigarrillo; sentimiento de que está perdiendo la razón; comportamientos rígidos, obstinación o terquedad y sensación de no poder manejar los problemas de la vida(44).

Los resultados de la aplicación fueron calificados de acuerdo con el manual del cuestionario del estrés y se analizaron desde la estadística descriptiva, utilizando el programa estadísticos informático Statistical Package for the Social Sciences (SPSS). Se usaron los baremos de jefes, profesionales y técnicos, que se reportan en el manual del usuario(44). Es de aclarar que el cuestionario no está diseñado para la obtención de resultados para cada uno de los síntomas y en tal sentido, como un aporte para determinar la prevalencia de cada síntoma en el resultado general del nivel de estrés; se realiza un proceso estadístico de manera tal, que los puntajes brutos, se transforme a una escala 0 a 100, para determinar el nivel de estrés, es decir si un individuo obtiene un puntaje bruto de 15 en síntomas fisiológicos, el puntaje transformado es:

$$
\frac{15}{25,5} \times 100=58,82
$$

Esta puntuación transformada de 58,82 indica que el síntoma fisiológico se encuentra en nivel Muy alto (Tabla 1).

Tabla 1. Baremos de la tercera versión del Cuestionario para la evaluación del estrés

\begin{tabular}{lr}
\hline \multicolumn{1}{c}{$\begin{array}{c}\text { Nivel de síntomas } \\
\text { de estrés }\end{array}$} & Puntaje total transformado \\
\hline Muy bajo & 0,0 a 7,8 \\
Bajo & 7,9 a 12,6 \\
Medio & 12,7 a 17,7 \\
Alto & 17,8 a 25,0 \\
Muy alto & 25,1 a 100 \\
\hline Nota: Se usó los baremos de Jefes - Profesionales - Técnicos. Manual \\
del Usuario(45)
\end{tabular}

\section{Consideraciones éticas}

La investigación privilegió la confidencialidad de los participantes y de las instituciones, para lo cual se firmó un consentimiento informado diseñado por los investigadores, y en tal sentido las consideraciones éticas se basa en los principios científicos, técnicos y administrativos establecidos para la investigación con seres humanos de acuerdo a la Ley 1090 del $2006^{(45)}$ y la Resolución 8430 de 1993(46). La investigación contó con el aval del Comité Ético de la institución financiadora.

\section{Resultados}

De los 595 participantes, el puntaje total transformado fue 21,7, lo que indica un nivel de estrés alto (Tabla 2). El nivel de estrés alto, indica que la cantidad de síntomas y su frecuencia de presentación son el reflejo de una respuesta de estrés severa y perjudicial para la salud, que requieren intervención en el marco de un sistema de vigilancia epidemiológica.

Tabla 2. Resultado general de la evaluación del estrés

\begin{tabular}{lrr}
\hline \multicolumn{1}{c}{ Síntomas } & $\begin{array}{c}\text { Puntaje total } \\
\text { transformado }\end{array}$ & $\begin{array}{c}\text { Nivel de } \\
\text { síntomas } \\
\text { de estrés }\end{array}$ \\
\hline Fisiológicos & 28,6 & Muy alto \\
Comportamiento social & 15,8 & Medio \\
Intelectuales y laborales & 20,6 & Alto \\
Psicoemocionales & 11,6 & Bajo \\
Total general estrés & 21,7 & Alto \\
\hline
\end{tabular}

Nota: Se usó los baremos de Jefes - Profesionales - Técnicos. Manual del Usuario ${ }^{(45)}$

En los síntomas fisiológicos, el puntaje total transformado de 28,6 ubica un nivel de estrés muy alto, lo que indica que la cantidad de síntomas y su frecuencia de presentación son indicativas de una respuesta de estrés severa y perjudicial para la salud, concretamente reportan dolores en el cuello y espalda o tensión muscular. En los síntomas de comportamiento social, el puntaje total transformado de 15,8 ubica un nivel de estrés medio, que evidencia ausencia de síntomas de estrés o escasa prevalencia, asociados con dificultad en las relaciones familiares.

En los síntomas intelectuales y laborales, el puntaje total transformado de 20,6 tiene un nivel de estrés alto, es decir hay prevalencia y frecuencia de los síntomas; concretamente reportan sentimiento de sobrecarga de trabajo, cansancio, tedio o desgano. En los síntomas 
psicoemocionales, el puntaje total transformado de 11,6 corresponde a un nivel de estrés bajo, indicando baja frecuencia de síntomas de estrés y por tanto sin afectación del estado general de salud, lo anterior se atribuye a bajo consumo de bebidas alcohólicas, café o cigarrillo y escaso sentimiento de angustia, preocupación o tristeza.

\section{Discusión}

En esta investigación se identificó nivel de estrés laboral alto en personal con cargos asistenciales de cuatro instituciones nivel III del sector salud de la ciudad de Cali, Colombia, encontrado en los resultados prevalencia de síntomas: fisiológicos caracterizados por tensión muscular, dolor de cuello y espalda, y síntomas intelectuales y laborales, que se manifiestan en una percepción de sobre carga de trabajo, cansancio, tedio o desgano.

Los síntomas fisiológicos obtenidos en este estudio, son semejantes a los reportados por Xu et al.(47), quienes encontraron relación entre casi todos los factores psicosociales con las enfermedades coronarias, neurológicas y psicológicas. López et al.(16), reportan ansiedad, tensión, cefaleas y problemas cardiovasculares como arritmias e hipertensión, además de los trastornos del sueño, la irritabilidad, depresión, fatiga y la acidez estomacal. Los estudios de Cesarini et al.(48) y Juárez(31), encontraron en los profesionales de la salud que sienten estar todo el día fatigados, con problemas para conciliar el sueño o aquellos que se despiertan sobresaltados durante el mismo.

En la investigación se encontró en el personal médico asistencial prevalencia de cefalea, diversos malestares del sistema músculoesquelético del sistema digestivo, agotamiento y tensión que normalmente se relacionan con estresores psicosociales del trabajo $y$ corresponden a síntomas fisiológicos(47), resultados similares a los obtenidos por Clausi et al.(49), donde un 22,22\% presentó consecuencias físicas derivadas del trabajo que realizan, como: dolores de cabeza, insomnio, dolores musculares. Estos síntomas físicos se pueden asociar con las condiciones laborales del sector salud(12) en donde la situación de emergencia, la sobrecarga entre la cobertura del servicio, que se expresa en número de pacientes y la polivalencia de las enfermedades conllevan a una demanda que en ocasiones sobrepasa las habilidades de las personas con cargos asistenciales en balance con la recompensa recibida que generan tensión laboral(29).

En la presente investigación los síntomas intelectuales y laborales en la presente investigación, se encontraron en nivel alto, caracterizado por sobrecarga de trabajo y dificultad para concentrarse u olvidos frecuentes, que se relacionan con disminución de la productividad o rendimiento laboral $y$ dinamismo(8,47). El trabajo en el sector salud implica realizar turnos y extensas jornadas diarias, lidiar con la enfermedad del paciente y la carga emoción al propia y de los familiares del paciente, situaciones que producen respuestas asociadas al estrés $(48,50)$. En particular el ser responsable de la seguridad y salud de otras personas desencadena estrés en los trabajadores de la salud(29), así como también la exposición permanente a las demandas emocionales de los pacientes y sus familiares que son condiciones propias del contexto de las instituciones de salud, lo que coincide con los hallazgos de Castillo et al.(6) y Vallejo et al.(51).

Estas condiciones laborales en Colombia se regulan por los lineamientos establecidos en la Ley 100 de 1993(3) y el Código Sustantivo de Trabajo(52), que determinó el trabajo en la modalidad de turnos rotarios; causando el pluriempleo y agotamiento físico en los trabajadores de la salud, que deben acudir a los turnos que no ofrecen la posibilidad de los descansos reparadores que se requieren para atender las demandas del sector que implica atención al detalle y la toma de decisiones conforme a las situaciones de urgencia $y$ emergencia(46).

La prevalencia de estrés laboral en el personal asistencial es de nivel alto, lo cual pone en evidencia que el estrés puede tener su génesis en las condiciones laborales, aunque su diagnóstico se puede diluir con enfermedades generales por 
la similitud de los síntomas fisiológicos, requiriendo un protocolo que responda a los lineamientos del Decreto 1477 de 2014(1), que define el estrés como enfermedad de origen laboral.

En la presente investigación se encontró relaciones en otros estudios, con factores psicosociales en enfermedades cardiovasculares, neurológicas y psicológicas(16), que sustentan la pertinencia de indagar desde diferentes perspectivas el impacto del estrés laboral en diferentes poblaciones y en particular en el de la salud, atendiendo a que el estrés es una enfermedad laboral que afecta el desempeño, satisfacción laboral y compromiso entre otros indicadores de índole organizacional. Es importante que las directivas de las instituciones de salud comprendan que las condiciones de trabajo propias del sector impactan la salud de sus trabajadores, para lo cual se debe iniciar por el diagnóstico del nivel de estrés identificando las causas que lo producen para orientar el tipo de intervención.

\section{Conclusiones}

Para enfrentar el estrés en las organizaciones de salud es necesario abarcar los niveles individual, grupal y organizacional. Capacitar al personal asistencial en atención en crisis, manejo de demandas emocionales, ofreciéndoles condiciones de trabajo que partan de los diseños de los puestos, claridad del rol, ajustes en los turnos de trabajo, políticas de recompensas en función del cumplimiento de indicadores, sistema de control de los riesgos psicosociales Por lo tanto, se hace necesario investigar más sobre el estrés como enfermedad laboral, para diseñar estrategias que disminuyan los síntomas de estrés a los que están expuestos, mejorando la salud y el bienestar integral de los profesionales de la salud teniendo en cuenta, el entorno organizacional propio del sector de la salud.

\section{Financiación}

Este artículo es derivado de la investigación registrada con el código $\mathrm{N}^{\circ}$ 020100358, financiada por la Pontificia Universidad Javeriana Cali.

\section{Conflicto de intereses}

Los autores declaran no tener conflicto de intereses en el desarrollo y divulgación de esta investigación.

\section{Referencias}

1. Ministerio del Trabajo. Decreto número 1477 de 2014 [Internet]. 2017. Available from: http://www.ins.gov.co/normatividad/Decretos/DECR ETO\%201477\%20DE\%202014.pdf

2. Ministerio del Trabajo. II Encuesta nacional de condiciones de seguridad y salud en el trabajo en el sistema general de riesgos laborales. 2013 [Internet]. 2017. Available from: http://ccs.org.co/salaprensa/images/Documentos/INF ORME_EJECUTIVO_II\%20ENCSST.pdf

3. Congreso de la República de Colombia. Ley número 100 de 1993 [Internet]. 2017. Available from: http://www.alcaldiabogota.gov.co/sisjur/normas/Nor ma1.jsp?i=5248

4. Díaz D. Estrés laboral y sus factores de riesgo psicosocial. Rev CES Salud Pública. 2010; 2 (1): 80-84.

5. Posada E. La relación trabajo - estrés laboral en los colombianos. Rev CES Salud Pública. 2011; 2 (1): 66-73.

6. Castillo I, Torres N, Ahumada A, Cárdenas K, Licona S. Estrés laboral en enfermería y factores asociados. Cartagena (Colombia) Rev Salud Uninorte; Barranquilla. 2014; 30 (1): 34-43.

7. Osorio J, Cárdenas L. Estrés laboral: estudio de revisión. Rev Diversitas. Perspectivas en psicología. 2017; 13 (1): 81-90.

8. Atalaya M. El estrés laboral y su influencia en el trabajo. Rev Industrial Data. 2001; 4 (2): 25-38.

9. Duran M. Bienestar psicológico: El estrés y la calidad de vida en el contexto laboral. Rev Nacional de Administración. 2010; 1 (1): 71-84.

10. Fernández J, Siegrist J, Rödel A, Hernández R. El estrés laboral: un nuevo factor de riesgo. ¿Qué sabemos y qué podemos hacer? Rev Aten Primaria. 2003; 31 (8): 1-10.

11. Ansoleaga E. Indicadores de salud mental asociados riesgo psicosocial laboral en un hospital público. Rev Med Chile. 2015; 143: 47-55.

12. Pozos E, Preciado M, Plascencia A. Síntomas psicofisiológicos predictores del estrés en inmigrantes mexicanos en Canadá. Rev Ansiedad y Estrés. 2015; 21 (2-3): 143-156.

13. Crespo M, Labrador F. Evaluación y tratamiento del Estrés. En: Buceta JM, Bueno AM, Mas B, editores. Intervención psicológica y salud: Control del estrés y conductas de riesgo. Madrid: Dykinson; 2001. p. 57-104.

14. Sosa EC. Qué es el estrés ocupacional, enfermedades derivadas y las reconocidas por la legislación colombiana. Rev CES Salud Pública. 2011; 2 (1): 56-65.

15. Villalobos G. Vigilancia epidemiológica de los factores psicosociales. Aproximación conceptual y valorativa. Rev Ciencia y Trabajo. 2004; 6 (14): 197-201.

16. López L, Solano A, Arias A, Aguirre S, Osorio C, Vásquez $\mathrm{M}$. El estrés laboral y los trastornos psiquiátricos en 
profesionales de la medicina. Rev CES Salud Pública. 2012; 3 (2): 280-288.

17. Lazarus RS, Folkman S, Folkman S. Estrés y procesos cognitivos [Internet]. Eds. Martínez Roca; 1986 [cited 2017 Jul 6]. Available from: https://dialnet.unirioja.es/servlet/libro?codigo $=22298$ 1

18. Kotekewis $\mathrm{K}$, Ribeiro $\mathrm{R}$, Ribeiro $\mathrm{B}$, Martins J. Enfermedades crónicas no transmisibles y el estrés de los trabajadores de enfermería de unidades quirúrgicas. Rev Enfermería Global. 2017; (46): 295-304.

19. Bakker A, Demerouti E. La teoría de las demandas y los recursos laborales. Journal of Work and Organizational Psychology. 2013; 29: 107-115.

20. Coffey M. Stress and burnout in forensic community mental health nurses: an investigation of its causes and effects. Rev J Psychiatr Ment Health Nurs. 1999; 6(6):433-443.

21. Oddie S, Ousley L. Assessing burn-out and occupational stressors in a medium secure service. Br J Forensic Pract [Internet]. 2007; Jul 11 [cited 2017 Jul 6]; 9(2):32-48. Available from: http://www.emeraldinsight.com/doi/10.1108/146366 46200700011.

22. Arenas J. Estrés en médicos residentes en una unidad de atención médica de tercer nivel. Rev Cirujano General. 2006; 28 (2): 103-109.

23. Cogollo M, Bustamante G. Condiciones laborales en enfermeras de Cartagena Colombia. Rev Av Enfer. 2010; 28 (1): 31-38.

24. Vinaccia S, Alvarán L. El síndrome del Burnout en una muestra de auxiliares de enfermería: un estudio exploratorio. Rev Terapia Psicológica. 2004; 22 (1): 916.

25. Cabrera A. Presentación. Directora General de Riesgos Profesionales- Ministerio de la Protección Social. Plan Nacional de Salud Ocupacional 2015. [Internet]. 2017. Available from:

http://www.minsalud.gov.co/Documentos\%20y\%20Public aciones/PLAN\%20NACIONAL\%20DE\%20SALUD\%200 CUPACIONAL.pdf.

26. Benavides F, Ruíz C, García A. Salud Laboral. Conceptos y Técnicas para la prevención de riesgos laborales. Barcelona: Editoral Masson; 2006.

27. Escriba-Agüir V, Artacoz L, Pérez-Hoyos S. Efecto del ambiente psicosocial y de la satisfacción laboral en el síndrome de burnout en médicos especialistas. Rev GacSanit. 2008; 22 (4): 300-308.

28. Eslava J, Barón G, Gaitán-Duarte H, Alfonso H, Agudelo C, Sánchez C. Evaluación del Impacto en Costo-equidad del Sistema de Salud en Colombia. Rev Salud Pública. 2008; 10 (1): 3-17.

29. Chiang M, Gómez N, Sigoña I. Factores psicosociales, stress y su relación con el desempeño: comparación entre centros de salud. Revi Salud Tra. 2013; 21 (2): 111-128.

30. Juárez A. Factores psicosociales, estrés y salud en distintas ocupaciones: un estudio exploratorio. Rev Investigación en Salud. 2007; 9 (1): 57-64

31. Messeguer M, Sánchez M, García M, Sáez M, Sánchez J. Los Factores psicosociales de riesgo en el trabajo como predictores del Mobbing. Rev Psicothema. 2007; 19, (2): 225-230.

32. Sevilla $\mathrm{T}$, Uribe $\mathrm{H}$, Rodríguez L. Una mirada a la construcción de género y el conflicto en tres entornos laborales en Cali. Cali: Editorial Universidad Autónoma de Occidente; 2010.

33. Gil-Monte P. Riesgos psicosociales en el trabajo y salud ocupacional. Rev Perú Med Exp Salud Pública. 2012; 29 (2): 237- 241.

34. Landa V, Mena L. Estrés laboral en personal clínico hospitalario. Rev Terapia Psicológica. 2003; 21 (2): 137 151.

35. Organización Mundial de la Salud. British Medical Journal. 2000; 320: 237-243. En: www.who.org.

36. Blanch J. Psicología Social del Trabajo en: Álvaro J, Garrido A, Torregrosa J. Psicología Social Aplicada. Barcelona: Mac Graw Hill; 1996. p. 155-198.

37. Rentería E. El modelo educativo tradicional y los perfiles de competencias según las modalidades y tendencias del trabajo actuales. España: Editorial AUIP; 2001.

38. Ancinas P. Burn-out y desgaste por empatía en profesionales de cuidados paliativos. Rev Digital de Medicina Psicosomática y Psicoterapia. 2012; 2 (4): 122.

39. Lepidou M, Kadda O, Argyriou G, Nestor A, Marvaki K, Hassioti G, Nanas S. Ethical dilemmas as a source of stress among doctors and nurses in the intensive care unit. Rev Nosileftiki. 2012; 51 (2): 187-194.

40. Schreuder J, Roelen C, Koopmans P, Moen B, Groothoff J. Effort-reward imbalance is associated with the frequency of sickness absence among female hospital nurses: A cross-sectional study. International Journal of Nursing Studies. 2010; 47 (5): 569-576.

41. Aniţei M, Stoica I, Samsonescu M. Particularities of personality traits and perceived stress at workplace. Rev For the young workers in Romania. 2013; 84: 10101014.

42. Ministerio de la Protección Social. Resolución número 2646 de 2008 [Internet]. 2017. Available from: http://www.alcaldiabogota.gov.co/sisjur/normas/Nor ma1.jsp?i=31607.

43. Villalobos G. Diseño de un sistema de vigilancia epidemiológica de factores de riesgo psicosocial en el trabajo. Tesis doctoral para la obtención del grado de Doctor en ciencias de la Salud. Escuela Nacional de Salud Pública, La Habana. 2005.

44. Ministerio de la Protección Social Batería de instrumentos para la evaluación de factores de riesgo psicosocial. Bogotá D.C.: Ministerio de la Protección Social. 2010.

45. Congreso de la República de Colombia. Ley 1090 de 2006 [Internet]. 2017. Available from: http://www.sociedadescientificas.com/userfiles/file/L EYES/1090\%2006.pdf

46. Ministerio de Salud. Resolución número 8430 de 1993 [Internet]. 2017. Available from:

https://www.minsalud.gov.co/sites/rid/Lists/BibliotecaDi gital/RIDE/DE/DIJ/RESOLUCION-8430-DE1993.PDF

47. Xu W, Hang J, Gao W, Zhao Y, Li W, Wang X, et al. Association between effort-reward imbalance and glycosylated hemoglobin (HbA1c) among Chinese 
workers: results from SHISO study. Int Arch Occup Environ Health. 2012; 85 (2): 215-220.

48. Cesarini J, Pagani J, Rodríguez A, Wainszelbaum N. El personal de salud, la actividad hospitalaria y la prevención del daño laboral. Boletín Científico, Asoc Med Municip de la CBA, Buenos Aires 2001. [Internet]. 2017. Available from: http://www.medicosmunicipales.org.ar/bc0601.htm.

49. Clausi A, Torales E, Czernik, G, Almirón L. Estrés asistencial y laboral en un Servicio Hospitalario de Clínica Médica de la ciudad de Corrientes. Rev Argentina de Clínica Neuropsiquiátrica. 2007; 13(4): 30-39.

50. Organización Mundial de la Salud. (2000). The World Health Report 2000. Health systems: Improving performance.

http://www.cdc.Gov/(spanish/niosh/docs/99101sp.htlm.

51. Vallejo B, Pérez A. Estrés Vital: variables psicológicas y sociodemográficas predictoras del malestar emocional. Rev Acción Psicológica. 2016; 13(1): 159-178.

52. Congreso de la República de Colombia. Decreto Ley 2663 del 5 de agosto de 1950. [Internet]. 2017. Available from:

http://www.alcaldiabogota.gov.co/sisjur/normas/Nor ma1.jsp?i=33104 